\title{
A Convenient Synthesis of Chiral Non-Racemic Vinyl Aziridines
}

Daniel Morton $^{\mp}$, David Pearson ${ }^{\ddagger}$, Robert A. Field $^{\mp}$ and Robert A. Stockman ${ }^{\mp *}$

${ }^{7}$ School of Chemical Sciences and Pharmacy, University of East Anglia, Norwich, NR4 7TJ, UK.Fax+44(1603)592003,*r.stockman@uea.ac.uk

${ }^{\ddagger}$ Millenium Pharmaceuticals UK, Granta Park, Great Abington, Cambridge, CB1 6ET, UK

\section{Contents:}

$\rightarrow$ General procedure for the preparation of tert-butylsulfinyl-vinyl-aziridines -

Page 2

$\rightarrow$ Experimental data for selected tert-butylsulfinyl-vinyl-aziridines -

Pages $2-6$

$\rightarrow$ Typical procedure for the de-protection of tert-butylsulfinyl-vinyl-aziridines -

Page 7

$\rightarrow$ Experimental data for hydrochloride salt of 3-phenyl-vinyl-aziridine -

Page 7 


\section{General procedure for the preparation of tert-butylsulfinyl-vinyl-aziridines}

A solution of the tetrahydrothiophene allyl sulfur salt, ( $1.5 \mathrm{eq}, 1.5 \mathrm{mmol}$ ), in anhydrous tetrahydrofuran, $(5 \mathrm{ml}$ ), was stirred at room temperature under an atmosphere of argon. After 10 minutes, a solution of the tert-butylsulfinylimine, ( $1 \mathrm{eq}, 1 \mathrm{mmol}$ ), in anhydrous tetrahydrofuran, ( $5 \mathrm{ml}$ ), was added to the reaction mixture. The cloudy dispersion was then stirred for a further 20 minutes. At this stage the lithium tert-butoxide, ( $1.5 \mathrm{eq}, 1.5 \mathrm{mmol}$ ), was added, portion-wise, to the reaction mixture, resulting in a significant colour change. Once the reaction was complete by TLC, ice-cold brine, ( $15 \mathrm{ml}$ ), was added, and the biphasic reaction was stirred rapidly for 10 minutes. The resulting cloudy mixture was then filtered through a pad of celite, the product extracted into diethyl ether, washed with brine and dried over sodium sulfate. The organic fraction was concentrated in vacuo to yield a crude mixture containing the azirdine. The desired products were isolated by column chromatography.

\section{tert-butylsulfinyl-cyclohexyl-vinylaziridine}

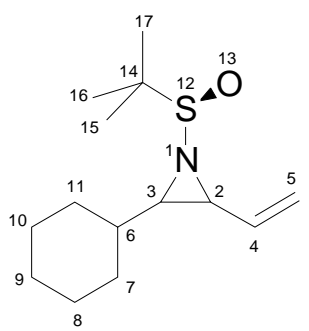

Isolated over neutral alumina, Pet. Ether / Ethyl acetate 15:1, as a viscous, colourless oil, $78 \%$. $[\alpha]_{\mathrm{D}}-$ 75 (c 1.0, $\mathrm{CHCl}_{3}$ ). IR: 2927, 2848, 2360, 1444, 1360, 1078. MS: m/z = [M+H] 256.2 (95), 154.2 (100). HRMS: Calcd for $\mathrm{C}_{14} \mathrm{H}_{26} \mathrm{NOS}[\mathrm{M}+\mathrm{H}]: 256.1730$. Found: $256.1731 . \delta_{\mathrm{H}}\left(400 \mathrm{MHz}, \mathrm{CDCl}_{3}\right)$, trans : 6.08 (1H, ddd, J 7.34, 17.09 and 9.91, H-4), $5.26(1 \mathrm{H}, \mathrm{d}, \mathrm{J}$ 17.03, H-5), 5.09 (1H, d, J 9.71, H-5), $2.82(1 \mathrm{H}$, dd, J 9.42 and 3.87, H-3), $2.36(1 \mathrm{H}, \mathrm{dd}, \mathrm{J} 7.33$ and 3.87, H-2), 1.82, (1H, m, H-6), $1.66(6 \mathrm{H}, \mathrm{m}, \mathrm{H}-7, \mathrm{H}-$ 9 and $\mathrm{H}-11), 1.19(9 \mathrm{H}, \mathrm{s}, \mathrm{H}-15$ to $\mathrm{H}-17), 1.13(4 \mathrm{H}, \mathrm{m}, \mathrm{H}-8, \mathrm{H}-10) ; \delta_{\mathrm{C}}\left(100 \mathrm{MHz}, \mathrm{CDCl}_{3}\right)$ trans 135.91, $120.45,57.40,50.20,47.50,26.43,25.97,25.83,25.77,23.56$. cis $132.47,118.71,56.97,39.66$, 36.52, 30.94, 30.41, 30.06, 29.97. 


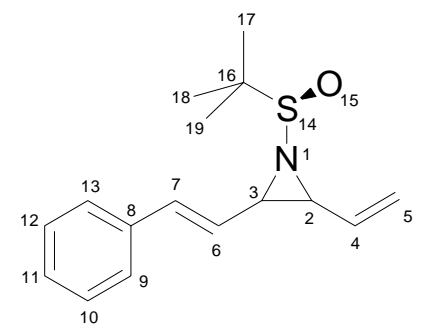

Isolated over neutral alumina, (Pet. Ether/ethyl acetate, 15:1), as a clear yellow oil, $82 \%$. $[\alpha]_{D}-50$ (C 0.5, $\mathrm{CHCl}_{3}$ ). IR: 2950, 2863, 1676, 1620, 1450, 1086. MS: $\mathrm{m} / \mathrm{z}=[\mathrm{M}+\mathrm{H}] 276.2$ (100). HRMS: Calcd for $\mathrm{C}_{16} \mathrm{H}_{22} \mathrm{NOS}[\mathrm{M}+\mathrm{H}]: 276.1417$. Found: $276.1418 . \delta_{\mathrm{H}}\left(400 \mathrm{MHz}, \mathrm{CDCl}_{3}\right)$ trans : $7.30(5 \mathrm{H}, \mathrm{m}, \mathrm{H}-9$ to $\mathrm{H}-$ 13), $6.70(1 \mathrm{H}, \mathrm{d}, \mathrm{J}$ 15.85, H-7), 6.26 (1H, dd, J 8.50, 15.80, H-6), 5.92 (1H, ddd, J 10.29, 16.98, 8.16, H-4) $5.45(1 \mathrm{H}, \mathrm{d}, \mathrm{J}$ 17.00, H-5), $5.29(1 \mathrm{H}, \mathrm{d}, \mathrm{J}$ 10.30, H-5), 3.19 (1H, dd, J 3.64, 8.49, H-3), $3.14(1 \mathrm{H}$, dd, J 3.70, 8.26, H-2), 1.27 (9H, s, $\mathrm{H}-17$ to $\mathrm{H}-19)$. $\delta_{\mathrm{C}}\left(100 \mathrm{MHz}, \mathrm{CDCl}_{3}\right)$ 23.28, 48.14, 48.67, 119.94, $125.64,126.66,128.17,128.84,134.21,134.56$.

\section{tert-butylsulfinyl-phenyl-vinylaziridine}

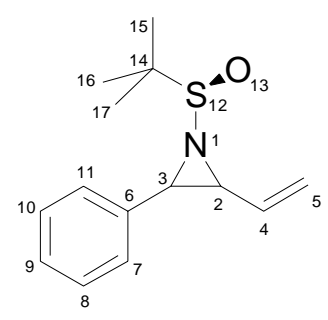

Isolated over neutral alumina, ( Pet. Ether/Ethyl acetate, 10:1), as a clear colourless oil, $68 \%$. $[\alpha]_{D}-95$ (c 1.0, $\mathrm{CHCl}_{3}$ ). IR: 3075, 2956, 2871, 1601, 1460, 1355, 1080. MS: $m / z=[\mathrm{M}+\mathrm{H}] 250.2$ (25), 228.2 (100). HRMS: Calcd for $\mathrm{C}_{14} \mathrm{H}_{12} \mathrm{NOS}[\mathrm{M}+\mathrm{H}]$ : 250.1265. Found: 250.1266. $\delta_{\mathrm{H}}\left(400 \mathrm{MHz}, \mathrm{CDCl}_{3}\right)$ trans : 7.24 (5H, m, H-7 to H-11), 6.20 (1H, ddd, J 16.97, 9.99, 9.51, H-4), 5.39 (1H, d, J 16.91, H-5), 5.28 (1H, d, J 10.25, H-5), $3.47(1 \mathrm{H}, \mathrm{d}, \mathrm{J} 3.55, \mathrm{H}-3), 3.09(1 \mathrm{H}, \mathrm{dd}, \mathrm{J} 3.59,9.41, \mathrm{H}-2), 1.21(9 \mathrm{H}, \mathrm{s}, \mathrm{H}-15$ to H17). $\delta_{\mathrm{C}}\left(100 \mathrm{MHz}, \mathrm{CDCl}_{3}\right) 20.55,36.50,48.10,54.98,119.57,124.45,125.58,126.38,130.36$. 


\section{tert-butylsulfinyl-napthyl-vinylaziridine}

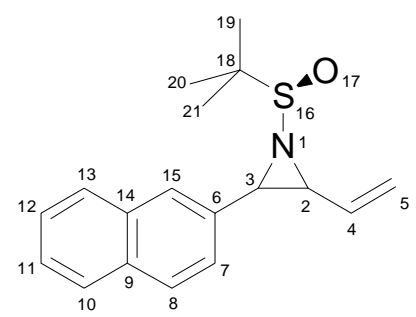

Isolated over silica, (Pet. Ether/Ethyl acetate, 10:1), as a colourless crystalline solid, $64 \%$. $[\alpha]_{D}-34$ (c 0.5, $\mathrm{CHCl}_{3}$ ). IR: 3060, 2980, 2860, 1601, 1500, 1474, 1361, 1076. MS: $m / z=[\mathrm{M}+\mathrm{H}] 300.3$ (82), 181.2 (100). HRMS: Calcd for $\mathrm{C}_{18} \mathrm{H}_{22} \mathrm{NOS}[\mathrm{M}+\mathrm{H}]$ : 300.1417. Found: $300.1414 . \delta_{\mathrm{H}}\left(400 \mathrm{MHz}, \mathrm{CDCl}_{3}\right)$ trans : $7.75(4 \mathrm{H}, \mathrm{m}, \mathrm{H}-10$ to $\mathrm{H}-13), 7.40(3 \mathrm{H}, \mathrm{m}, \mathrm{H}-7, \mathrm{H}-8$ and $\mathrm{H}-15), 6.24(1 \mathrm{H}$, ddd, J 9.50, 10.13, 17.16, H4), $5.43(1 \mathrm{H}, \mathrm{d}, \mathrm{J}$ 18.00, H-5), $5.31(1 \mathrm{H}, \mathrm{d}, \mathrm{J}$ 10.31), $3.62(1 \mathrm{H}, \mathrm{d}, \mathrm{J} 3.63, \mathrm{H}-3), 3.20(1 \mathrm{H}, \mathrm{dd}, \mathrm{J} 3.51$, 9.38), $1.18(9 \mathrm{H}, \mathrm{s}, \mathrm{H}-19$ to $\mathrm{H}-21)$. cis : $4.05(1 \mathrm{H}, \mathrm{d}, \mathrm{J} 7.02, \mathrm{H}-3), 3.03(1 \mathrm{H}, \mathrm{t}, \mathrm{J} 7.18, \mathrm{H}-2) . \delta_{\mathrm{C}}(100 \mathrm{MHz}$, $\left.\mathrm{CDCl}_{3}\right), 22.81,38.01,52.02,57.29,121.92,123.91,126.05,126.39,127.75,128.53,132.58,133.06$, 133.26 .

\section{tert-butylsulfinyl-hexyl-vinylaziridine}

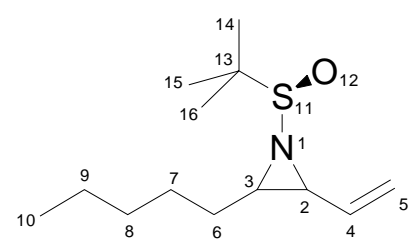

Isolated over neutral alumina, (Pet. Ether/Ethyl acetate, 15:1), as a clear colourless, viscous oil, 67\%. $[\alpha]_{\mathrm{D}}-25\left(\mathrm{c} 0.5, \mathrm{CHCl}_{3}\right) . \mathrm{IR}: 2955,2852,1650,1454,1086 \mathrm{~cm}^{-1} . \mathrm{MS}: \mathrm{m} / \mathrm{z}=[\mathrm{M}+\mathrm{H}] 244.3$ (98), 142.2 (100). HRMS: Calcd for $\mathrm{C}_{13} \mathrm{H}_{26} \mathrm{NOS}[\mathrm{M}+\mathrm{H}]: 244.1730$. Found: 244.1734. $\delta_{\mathrm{H}}\left(400 \mathrm{MHz}, \mathrm{CDCl}_{3}\right)$, trans: $5.92(1 \mathrm{H}, \mathrm{ddd}, \mathrm{H}-4), 5.30(1 \mathrm{H}, \mathrm{d}, \mathrm{H}-5), 5.18(1 \mathrm{H}, \mathrm{d}, \mathrm{H}-5), 2.71(1 \mathrm{H}, \mathrm{dd}, \mathrm{H}-2), 2.45(1 \mathrm{H}, \mathrm{m}, \mathrm{H}-3), 1.75$ $(1 \mathrm{H}, \mathrm{m}), 1.5 \rightarrow 1.25(7 \mathrm{H}, \mathrm{m}), 1.18(9 \mathrm{H}, \mathrm{s}, \mathrm{H}-14$ to $\mathrm{H}-16), 0.90(3 \mathrm{H}, \mathrm{t}, \mathrm{H}-10) . \delta_{\mathrm{C}}\left(100 \mathrm{MHz}, \mathrm{CDCl}_{3}\right)$, $14.50,23.20,27.2,31.9,36.2,44.8,49.90,57.2,119.1,135.8$. 


\section{tert-butylsulfinyl-2-pyridyl-vinylaziridine}

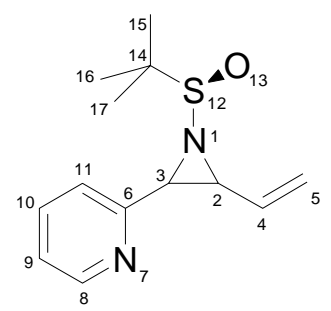

Isolated over neutral alumina, (Pet. Ether/Ethyl acetate, 10:1), as a pale yellow, very viscous oil, 54\%. $[\alpha]_{D}-22\left(\mathrm{c} 0.5, \mathrm{CHCl}_{3}\right) . \mathrm{MS}: \mathrm{m} / \mathrm{z}=[\mathrm{M}+\mathrm{H}] 251.1$ (25), 161.0 (100). HRMS: Calcd for $\mathrm{C}_{13} \mathrm{H}_{19} \mathrm{~N}_{2} \mathrm{OS}$ $[\mathrm{M}+\mathrm{H}]:$ 251.1213. Found: 251.1216. $\delta_{\mathrm{H}}\left(400 \mathrm{MHz} ; \mathrm{CDCl}_{3}\right) 8.52(1 \mathrm{H}, \mathrm{d}, \mathrm{J} 3.6, \mathrm{H}-8), 7.60(1 \mathrm{H}, \mathrm{dd}, \mathrm{J} 8$, 12.5, H-10), 7.27 (1H, d, J 8, H-11), 7.13 (1H, dd, J 3.6, 12.5, H-9), $5.51(1 \mathrm{H}, \mathrm{m}, \mathrm{H}-4), 5.37(1 \mathrm{H}, \mathrm{d}, \mathrm{J}$ 17.2, H-5), 5.15 (1H, d, J 10, H-5), 4.04 (1H, d, J 7.2, H-3), 3.06 (1H, dd, J 7.2, 14.8, H-2), $1.19(9 \mathrm{H}, \mathrm{s}$, $\mathrm{H}-15 \rightarrow \mathrm{H}-17) . \delta_{\mathrm{C}}\left(100 \mathrm{MHz} ; \mathrm{CDCl}_{3}\right)$ 154.81, 149.82, 136.43, 131.20, 122.73, 122.57, 121.23, 57.35, $42.04,39.33,22.98$.

\section{tert-butylsulfinyl-2-cyclopropyl-vinylaziridine}

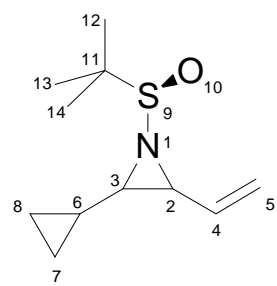

Isolated over neutral alumina, (Pet. Ether / Ethyl acetate 25:1), as a very pale yellow, quite fluid oil, 61\%. $[\alpha]_{D}-188$ (c 0.5, $\mathrm{CHCl}_{3}$ ). IR: 2957, 2920, 2850, 1457, 1361, 1080, 1020. MS: $m / z=[\mathrm{M}+\mathrm{H}] 214.2$ (100\%), HRMS: Calcd for $\mathrm{C}_{11} \mathrm{H}_{20} \mathrm{NOS}[\mathrm{M}+\mathrm{H}]:$ 214.1260. Found: 214.1262. $\delta_{\mathrm{H}}\left(400 \mathrm{MHz} ; \mathrm{CDCl}_{3}\right)$ trans: 5.96 (ddd, 1H, J 9.00, 9.99, 17.10, H-4), 5.36 (d, 1H, J 17.10, H-5), 5.20 (d, 1H, J 9.99, H-5), 2.84 (dd, $1 \mathrm{H}, \mathrm{J} 3.80,9.00, H-2), 2.43$ (dd, $1 \mathrm{H}, \mathrm{J} 3.80,5.66, H-3), 1.25$ (s, 9H, ${ }^{t}$ Butyl-H), 1.07 (m, $\left.1 \mathrm{H}, H-6\right), 0.56$ $(\mathrm{m}, 2 \mathrm{H}, \mathrm{H}-7), 0.34(\mathrm{~m}, 2 \mathrm{H}, \mathrm{H}-8) . \delta_{\mathrm{C}}\left(100 \mathrm{MHz} ; \mathrm{CDCl}_{3}\right)$ 132.20, 116. 40, 54.36, 46.11, 39.06, 27.04, $20.33,20.04,7.47$ 


\section{General procedure for the de-protection of tert-butylsulfinyl-vinyl-aziridines}

To a solution of tert-butylsulfinyl-vinyl-aziridine, ( 1 eq ), in anhydrous 1,4-dioxan, was added a solution of 1,4-dioxan saturated with $\mathrm{HCl}$ gas. The reaction was constantly monitored by $\mathrm{TLC}$, and the amount of $\mathrm{HCl}$ in solution was determined by the extent of reaction progress. Once all the starting material had been de-protected the solution was concentrated to a clear oil. The oil was then washed with diethyl-ether, yielding the $\mathrm{N}-\mathrm{H}$ aziridine as a white precipitate.

\section{3-phenyl-vinyl-aziridine}

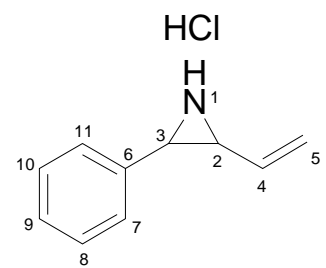

Isolated by washing with diethyl-ether, $(5 \times 10 \mathrm{ml})$, as a white solid precipitate, $>95 \%$ yield. $\mathrm{MS}: \mathrm{m} / \mathrm{z}=$ $[\mathrm{M}+\mathrm{H}]\left({ }^{35} \mathrm{Cl}\right) 182.1$ (30\%), 146.1 (100\%), 129.1 (48\%). HRMS: Calcd for $\mathrm{C}_{10} \mathrm{H}_{13} \mathrm{NCl}^{35}[\mathrm{M}+\mathrm{H}]: 182.0731$. Found: 182.0730. $\delta_{\mathrm{H}}\left(40 \mathrm{MHz} ; \mathrm{CD}_{4} \mathrm{OD}\right) 7.39(\mathrm{~m}, 5 \mathrm{H}, \mathrm{Ar}-\mathrm{H}), 5.89$ (ddd, $1 \mathrm{H}, \mathrm{J}$ 7.85, 10.55, 17.28, H-4), 5.49 (d, 1H, J 10.55, H-5), 5.35 (d, 1H, J 4.20, H-3), 5.27 (d, 1H, J 17.28, H-5), 4.23 (dd, 1H, J 4.20, 7.85, H-2). $\delta_{\mathrm{C}}\left(100 \mathrm{MHz} ; \mathrm{CD}_{4} \mathrm{OD}\right) 129.20,128.66,127.76,123.40,94.96,62.69,59.22$. 